\title{
SAND99.0106C
}

\section{COUPLED SIMULATIONS OF MECHANICAL DEFORMATION AND MICROSTRUCTURAL EVOLUTION USING POLYCRYSTAL PLASTICITY AND MONTE CARLO POTTS MODELS}

\author{
C.C. BATTAILE, T.E. BUCHHEIT, E.A. HOLM, G.W. WELLMAN, AND M.K. NEILSEN \\ Sandia National Laboratories, Albuquerque, NM 87185-1411, ccbatta@sandia.gov
}

\begin{abstract}
The microstructural evolution of heavily deformed polycrystalline $\mathrm{Cu}$ is simulated by coupling a constitutive model for polycrystal plasticity with the Monte Carlo Potts model for grain growth. The effects of deformation on boundary topology and grain growth kinetics are presented. Heavy deformation leads to dramatic strain-induced boundary migration and subsequent grain fragmentation. Grain growth is accelerated in heavily deformed microstructures. The implications of these results for the thermomechanical fatigue failure of eutectic solder joints are discussed.
\end{abstract}

\section{INTRODUCTION}

Thermal fluctuations during the normal course of service or storage can accelerate the failure of solder joints in electronic components [1]. This thermomechanical fatigue (TMF) of solder joints involves a number of different processes acting in concert. Thermal expansions and contractions of the surrounding pieces generate a complex state of stress in the joint. The local details of that stress state, and the properties of the solder microstructure, create regions of high localized strain in the joint. These localized deformations induce coarsening in the microstructure, which weakens the material. These weakened regions fatigue as the thermal fluctuations continue, eventually leading to component failure. This phenomenon has been observed, for example, as satellites pass into and out of the sun's rays, or when stored components are subjected to daily and seasonal temperature changes.

Understanding TMF requires an examination of how each process operates individually and in conjunction with the other processes. In this paper, we examine the effects of the localized deformation on the evolution of a polycrystal microstructure. Solder joints are typically composed of eutectic $\mathrm{PbSn}$, but we will use pure polycrystalline $\mathrm{Cu}$ as a proof-of-concept system for the present study. The deformation of the material is simulated using the finite element method (FEM) with a constitutive law for polycrystal plasticity fit to experimental data for $\mathrm{Cu}$. This polycrystal plasticity model predicts the local mechanical state of the system, and this information is combined with the Monte Carlo Potts model to simulate grain growth (i.e., microstructural coarsening) in the deformed material. The polycrystal plasticity and Potts models will be outlined briefly, and the extension of the Potts model to account for stored strain energies will be discussed. The effects of deformation on the evolution of the microstructure will be presented, and the implications of these results for the TMF of solder joints will be addressed.

\section{SIMULATION METHODS}

\section{Polverystal Plasticity}

The polycrystal plasticity model will be described here only in brief, and the reader is referred elsewhere $[2,3]$ for a more detailed discussion. The local states of stress and strain in the $\mathrm{Cu}$ polycrystal are simulated using FEM with a constitutive law for polycrystal plasticity fit to experimental data for $\mathrm{Cu}$. The finite element mesh is generated by discretizing a polycrystal microstructure onto a cubic lattice. Each lattice site is associated with a spatial orientation, and a cluster of lattice sites with the same orientation represents a grain. Each lattice site represents an element in the finite element mesh, and the nodes of the mesh form a cubic grid occupying the corners of the elements. Ideally, the polycrystal microstructure would correspond directly to the real $\mathrm{Cu}$ sample to which the plasticity model was fit. However, for the purpose of the present study, the microstructure was taken from a conventional 3D Potts model simulation, and the 


\section{DISCLAIMER}

This report was prepared as an account of work sponsored by an agency of the United States Government. Neither the United States Government nor any agency thereof, nor any of their employees, make any warranty, express or implied, or assumes any legal liability or responsibility for the accuracy, completeness, or usefulness of any information, apparatus, product, or process disclosed, or represents that its use would not infringe privately owned rights. Reference herein to any specific commercial product, process, or service by trade name, trademark, manufacturer, or otherwise does not necessarily constitute or imply its endorsement, recommendation, or favoring by the United States Government or any agency thereof. The views and opinions of authors expressed herein do not necessarily state or reflect those of the United States Government or any agency thereof. 


\section{DISCLAIMER}

Portions of this document may be illegible in electronic image products. Images are produced from the best available original document. 
orientations of the grains are assigned at random.

The constitutive model for the mechanical response of a polycrystal tracks the spatial orientations of the material at each element, and the material deformations on each of the twelve FCC slip systems. The elastic response of the material is anisotropic, and the plastic response is strain rate dependent such that $\tau \propto \dot{\gamma}^{m}$, where $\tau$ is the shear stress, $\dot{\gamma}$ is the shear strain rate, and $m$ is the strain rate sensitivity exponent. In this study, an exponent of $m=10$ was used. Each of the twelve slip systems hardens isotropically according to a power law,

$$
d \tau=\tau_{o}+A d \gamma^{n}
$$

where $\tau_{o}$ is the initial critical resolved shear stress, $\gamma$ is the effective plastic shear strain, and $A$ and $n$ are parameters fit to the experimental stress-strain response of a Cu polycrystal. All slip systems are initialized to the same critical resolved shear stress and harden equally. However, the polycrystal evolves an anisotropic mechanical response during deformation due to the variations in geometric constraints between and within grains.

The plasticity simulation is performed on a 54-grain polycrystal microstructure mapped onto a $30 \times 30 \times 30$-element mesh. The average grain size is about 500 elements. Each element is cubic before deformation. The initial element orientations in each grain are the same (random) value, and the stress and strain values at each element set to zero. Periodic boundary conditions are imposed in all three directions. Deformation is simulated by a prescribed elongation of the simulation cell in one direction, with zero net force in the other two directions. The elongation of the simulation cell is increased in increments of $0.001 \%$. At each strain increment, the mechanical state of the system is equilibrated. The stress and strain increments on each slip system in each element are used to calculate the stored plastic energy, which is input to a Monte Carlo Potts model of microstructure evolution, as described below. (Stored elastic energy is relatively small at the deformations of interest, and is therefore neglected in the present study, though it may be important under certain circumstances.)

\section{Microstructural Evolution}

The evolution of the deformed microstructures is simulated using a Monte Carlo Potts model $[4,5]$ modified to account for the plastic energy stored in the material during deformation. The finite element mesh is input directly to the Potts simulation. Each grain on the mesh is assigned a unique integer identity, or "spin," and all the elements in a grain assume the spin of that grain. Periodic boundary conditions are imposed in all directions. Each element contains twelve slip systems for which the twelve shear stress and strain increments are taken from the polycrystal plasticity model.

The system energy is described by a modified Potts Hamiltonian,

$$
H=\sum_{i=1}^{N}\left\{\frac{E_{o}}{2} \sum_{j=1}^{26}\left[1-\delta\left(s_{i}, s_{j}\right)\right]+\frac{1}{V_{i} \tau_{o}} \sum_{\Delta l=0 \alpha=1}^{\Delta l_{j}} \sum_{i}^{12} \tau_{i}(\alpha, \Delta l) \Delta \gamma_{i}(\alpha, \Delta l)\right\} .
$$

The sum over $i$ includes all of the $N$ elements in the mesh, and the sum over $j$ includes all of the 26 elements that share at least one node with element $i$. (Recall that the mesh is initially cubic, and thus the element connectivity of the deformed mesh is that of a cubic grid.) The sum over $\Delta l$ includes all elongation increments up to the elongation of interest, $\Delta l_{f}$, and the sum over $\alpha$ includes all twelve slip systems in element $i . E_{o}$ is a measure of the grain boundary energy, and is assumed to be unity for all boundaries in this study. $s_{i}$ is the spin of element $i$, and $\delta\left(s_{i}, s_{i}\right)$ is the Kronecker delta, i.e., $\delta\left(s_{i}, s_{j}\right)=1$ if $s_{i}=s_{j}$ and 0 otherwise. $V_{i}$ is the volume of element $i$, $\tau_{j}$ is the initial critical resolved shear stress, $\tau_{i}(\alpha, \Delta l)$ is the average plastic shear stress on slip system $\alpha$ in element $i$ at elongation $\Delta l$, and $\Delta \gamma_{i}(\alpha, \Delta l)$ is the plastic shear strain increment on slip system $\alpha$ in element $i$ at elongation $\Delta l$. The first term in Equ. 2 represents the grain boundary energy, and the second describes the stored plastic energy.

The Monte Carlo Potts simulation proceeds by choosing an element at random and attempting to change its spin to that of one of its neighbors. When an element changes its spin to 
that of a different grain, the stored plastic energy in that element is changed to the average of the values at the neighboring elements that belong to that different grain. This implies that, when a grain boundary moves, nearly all of the dislocations adjacent to the boundary are extended into the material through which the boundary migrates. However, this is not always the case in reality. On the other hand, if no dislocations were propagated with the boundary, then a new grain would be introduced and the material would recrystallize. Though recrystallize undoubtedly occurs, and is certainly important in the materials of interest and at these levels of deformation, it is not the only option. A grain boundary can simply migrate in a heavily deformed polycrystal [6]. The migration of a grain boundary in a deformed polycrystal involves the propagation of some, but not always all, of the dislocations that are adjacent to it. However, a meaningful quantitative description of how the dislocation structure propagates with a migrating grain boundary is not currently available, and thus we assume that nearly all the dislocations are carried with a boundary when it moves.

The change to the system energy that results from an attempted spin "flip" is computed using Equ. 2, and the spin flip is accepted if the energy change is less than or equal to zero, and rejected otherwise. This is repeated until no more energy-decreasing spin flips are available. This pseudo-kinetic approach corresponds to the zero-temperature Metropolis Monte Carlo algorithm [7], though in practice we use a more efficient version $[8,9]$. The evolution of the microstructure (without deformation) toward its equilibrium reproduces the kinetics of normal grain growth remarkably well [10].

\section{RESULTS AND DISCUSSION}

The temporal evolution of the microstructure after $31 \%$ elongation is shown pictorially in Fig. 1. The black lines denote the grain boundaries. The shading corresponds to the stored plastic energy in each element, with dark regions representing low stored energy values and light regions indicating high values. As evident in Fig. 1, the grain boundaries roughen as they migrate and the grains fail to remain compact. This phenomenon is also observed experimentally [6] in deformed polycrystals, and is commonly termed strain-induced boundary migration (SIBM). SIBM occurs when two adjacent grains have very different dislocation densities (i.e., stored plastic energies). If the difference is much larger than the grain boundary energy, then the only significant driving force for grain boundary migration is the reduction in stored energy provided by the consumption of high-strain material by adjacent low-strain grains. Under these circumstances, grains are not motivated to remain compact and grain boundaries roughen, as seen in Fig. 1 and observed experimentally [6].
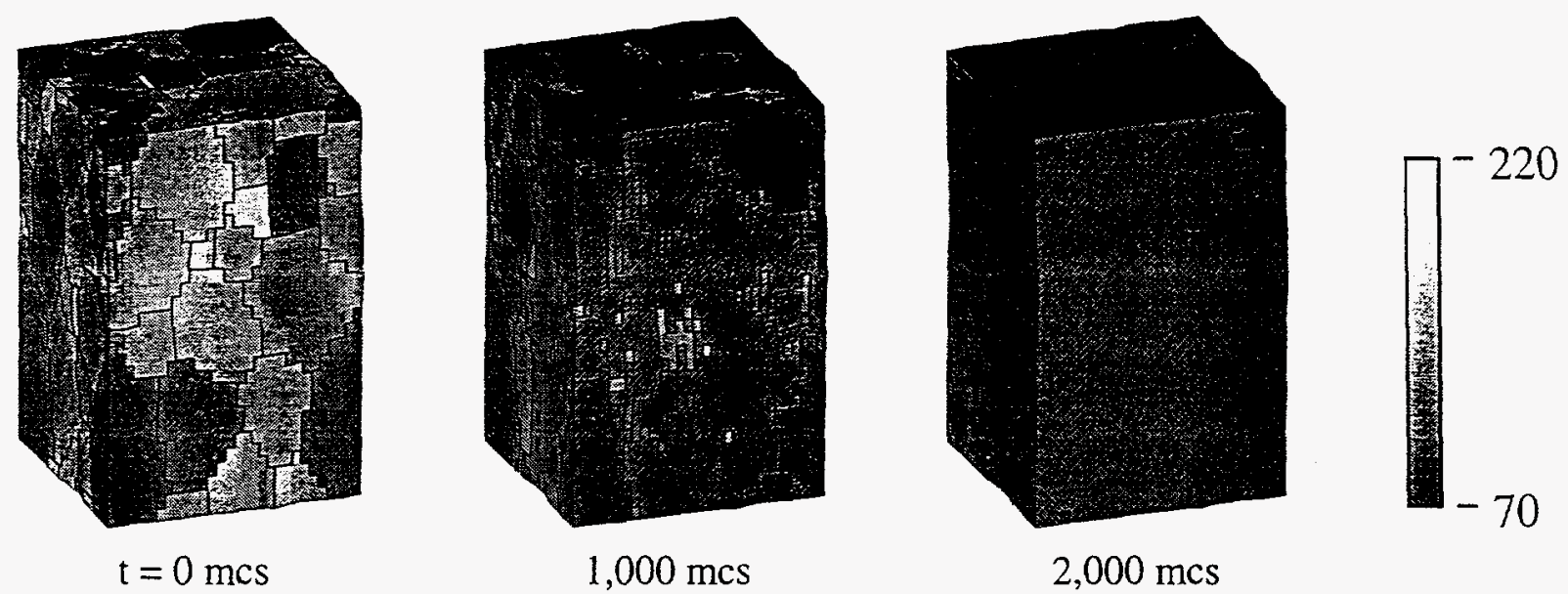

Figure 1. Evolution of the stored plastic energy during grain growth after $31 \%$ elongation. The stored energy distribution is shown at three simulation times in Monte Carlo steps (mcs). Dark areas have low stored energy and light areas have high energy. The scale bar at the right indicates normalized strain energy densities as per the second term in Equ. 2. 
At elongations larger than $31 \%$, SIBM becomes even more pronounced. At lower elongations, grains remain compact and the evolution of the microstructure more closely resembles normal grain growth, but boundary motion is still influenced by the stored energy in the material. This strain-enhanced boundary migration (SEBM) occurs when the driving forces due to grain boundary energy and stored plastic energy are comparable. This situation applies at $11 \%$ elongation, as depicted in Fig. 2, where grain growth appears normal but is dominated by material with relatively little stored plastic energy.

The grains that dominate the growth process at higher deformations aren't always the ones with the least stored plastic energy, since the plastic driving force for grain growth relies on the differences in stored energy between adjacent grains, and not on the absolute value of the stored energy. Therefore, the large grain in the last frame of Fig. 1 is lighter in shading (i.e., higher in stored plastic energy) than the grains that it surrounds. The same can be true for lower deformations, except that the significance of the curvature driving force (represented by the first term in Equ. 2) complicates the issue further.

By examining the time values in Figs. 1 and 2, one can readily see that the $31 \%$ elongated polycrystal coarsens much more quickly (by an order of magnitude) than does the $11 \%$ elongated structure. This is shown graphically in Fig. 3 which contains plots of the grain size evolution and the time required to reach an average grain radius of 10 elements, for various elongations. At large elongations in Fig. 3a, the average grain size decreases at early times as SIBM causes grains become non-compact and to fragment. At later times, the rate of grain growth increases with increasing elongation, so that the time required to achieve an average grain radius of ten elements decreases with increasing elongation, as in Fig. $3 \mathrm{~b}$.

Post mortem analyses of failed solder joints suggests that accelerated coarsening in regions of high local deformation leads to mechanical weakening and eventually failure of the joint [1]. The results presented in Fig. 3 support a part of this evidence by indicating that coarsening in singlephase polycrystals can be dramatically accelerated by deformation. As mentioned above, the plastic response of the simulated polycrystal is modeled after pure $\mathrm{Cu}$. However, the material used in many real-world components is eutectic $\mathrm{PbSn}$ solder. Coarsening in eutectics generally involves interphase diffusion, which cannot be captured by a model of single-phase grain growth.

Therefore, these results should be taken primarily as a demonstration of the modeling approach.

\section{SUMMARY}

Microstructural evolution in a 54-grain region of a deformed $\mathrm{Cu}$ polycrystal was simulated by coupling a constitutive finite element model of polycrystal plasticity with the Monte Carlo Potts model of grain growth. Heavy deformation introduces high stored plastic energies into the material which dominate the grain growth process. In this regime, grains are not motivated to remain compact, and strain-induced boundary motion leads to grain boundary roughening and grain

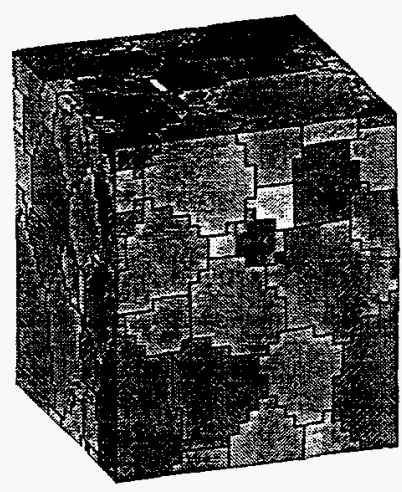

$\mathrm{t}=0 \mathrm{mcs}$

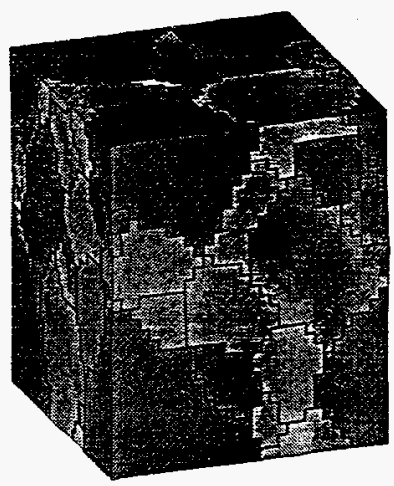

$10,000 \mathrm{mcs}$
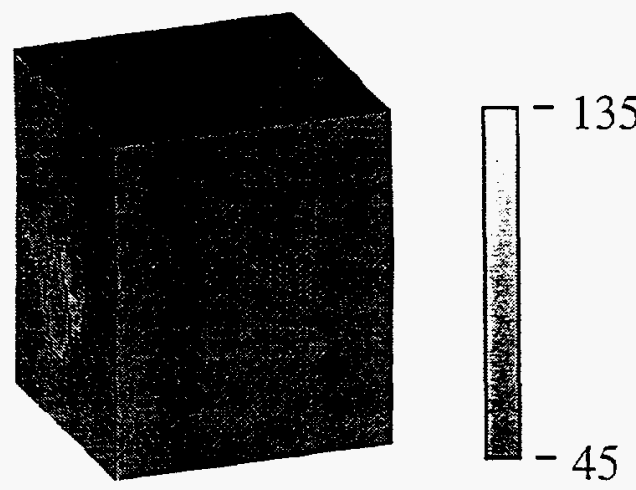

$20,000 \mathrm{mcs}$

Figure 2. Evolution of the stored plastic energy during grain growth after $11 \%$ elongation. The stored energy distribution is shown at three simulation times in Monte Carlo steps (mcs). Dark areas have low stored energy and light areas have high energy. The scale bar at the right indicates normalized strain energy densities as per the second term in Equ. 2. 


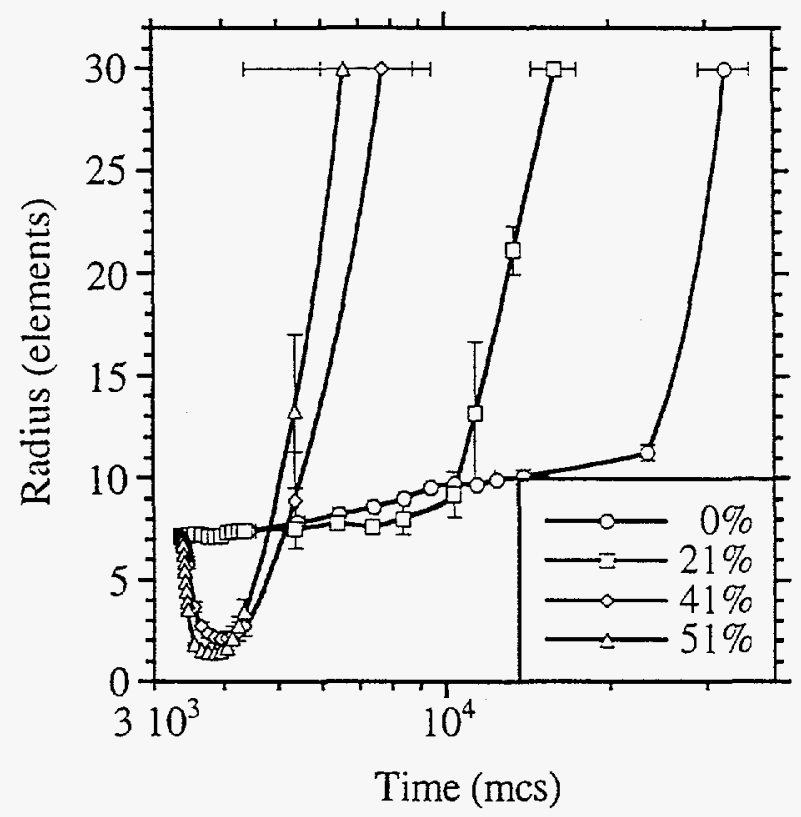

(a)

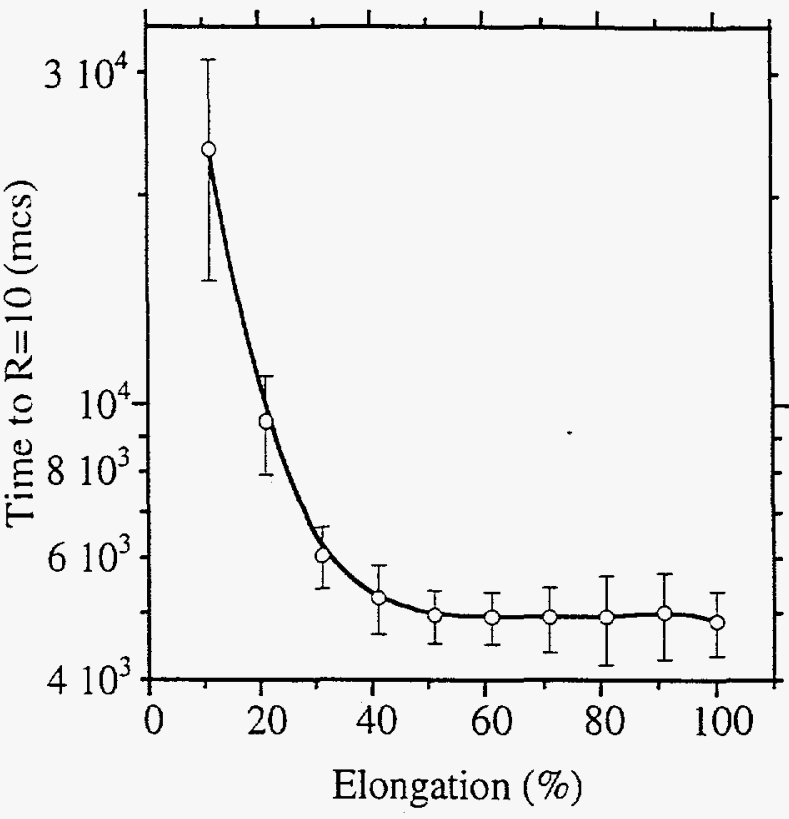

(b)

Figure 3. Grain growth kinetics: a) the average grain radius as a function of Monte Carlo time for four elongation values, and $b$ ) the Monte Carlo time required to achieve an average grain radius of ten elements as a function of the elongation. Error bars correspond to standard deviations of forty grain growth simulations at each elongation.

fragmentation. The additional driving force of the stored plastic energy serves to increase the coarsening rate with increasing deformation. This provides some insight into the thermomechanical fatigue failure of solder joints, which is precipitated by accelerated coarsening in locally deformed regions of the microstructure.

\section{ACKNOWLEDGMENTS}

This work was performed at Sandia National Laboratories under US DOE ContraztDERACO4 94AL85000.

\section{REFERENCES} Lerated by Sandia Cormoration, a Lockheed Marin Company. for the United States Department of Energy under contract DE-ACO4-94.AL85000.

[1] D.R. Frear, in Solder Mechanics: A State of the Art Assessment, edited by D.R. Frear, W.B. Jones, and K.R. Kinsman (TMS, Warrendale, PA, 1991), pp. 191-237.

[2] T.E. Buchheit, R.J. Bourcier, G.W. Wellman, and M.K. Neilsen, Mater. Sci. Eng. 5, pp. 421-437 (1997)

[3] U.F. Kocks, C.N. Tom, and H.-R. Wenk, Texture and Anisotropy: Preferred Orientations in Polycrystals and Their Effect on Materials Properties. 1998, Cambridge University Press: New York, NY. p. 676.

[4] M.P. Anderson, D.J. Srolovitz, G.S. Grest, and P.S. Sahni, Acta Metall. 32, pp. 783-91 (1984).

[5] D.J. Srolovitz, M.P. Anderson, P.S. Sahni, and G.S. Grest, Acta Metall. 32, pp. 793-802 (1984).

[6] P.A. Beck, in Metal Interfaces, edited by R.M. Brick (ASM, Cleveland, OH, 1952), pp. 208-47.

[7] N. Metropolis, A.W. Rosenbluth, M.N. Rosenbluth, A.H. Teller, and E. Teller, J. Chem. Phys. 21, pp. 1087-92 (1953).

[8] A.B. Bortz, M.H. Kalos, and J.L. Lebowitz, J. Comp. Phys. 17, pp. $10-8$ (1975).

[9] G.N. Hassold and E.A. Holm, Comp. in Phys. 7, pp. 97-107 (1993).

[10] M.P. Anderson, G.S. Grest, and D.J. Srolovitz, Phil. Mag. B 59, pp. 293-329 (1989). 